\title{
Web based tools for Road Traffic Monitoring and Controlling System
}

\author{
Camelia Avram ${ }^{1, a}$, Adina Aştilean ${ }^{1, b}$ and Radu Miron ${ }^{1, c}$ \\ ${ }^{1}$ Technical University of Cluj Napoca, Automation and Computer Science Faculty, Department of \\ Automation, Cluj Napoca, Romania

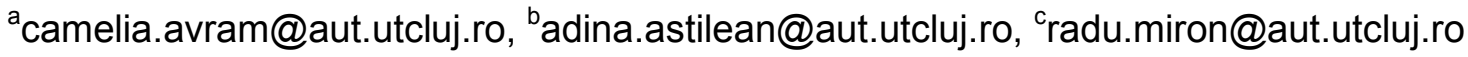

Keywords: road traffic, web tools, data fusion, modeling and simulation

\begin{abstract}
The Road Traffic Monitoring and Control System (RTMCS) presented in this paper is intended to offer support for the surveillance, control and monitoring of road networks. It integrates complex components in a modular, flexible and open structure in order to validate models that take into account real time constraints and include tools to simulate various traffic scenarios and communication technologies, WEB based virtual instrumentation, personalized user interfaces and relational data. The system can be used to change the configuration of the road map (to design new streets segments) or to visualize the results of the different traffic scenarios implying the tuning of various parameters. RTMCS also offers several advantages, one of the most important consisting of the possibility to choose different configuration and components using a web browser. An implementation variant, including suitable, promising new technologies, different maps configurations, communication devices and protocols and routing systems is presented and analyzed.
\end{abstract}

A large set of experiments and the corresponding results highlight the functionality of the configurable virtual web instrument concept illustrated by the proposed system.

Aspects regarding the possibility of a subsequent integration of new tools are also presented.

\section{Introduction}

The accuracy of the monitoring of traffic flow conditions, the design of realistic traffic simulators and appropriate traffic control strategies are the most important factors that could influence the performances of traffic management systems. New trends and perspective solutions in this field include the use of VANET's applications and Web-based tools to find efficient solutions and to manage, analyze and evaluate control strategy changes.

In [1] authors develop an application of the virtual instrument technology and use concepts of traffic flow theory, multi-sensor data fusion technology, database and fault diagnosis system in order to obtain a more detailed traffic condition information such as traffic volume, average velocity, link travel time, occupancy, etc. An application based on mobile technologies is proposed in [2]. Authors implement an Android-based mobile device platform that successfully programs a CAN-Bus driver which reads the data on the ECUs (Engine Control Unit) of the vehicle. Using virtual instruments, the results are graphically displayed on the mobile panel.

A Web Based Sensors concept and a system of wireless, intercommunicating, spatially distributed sensors tags that can be easily deployed in new environments for monitoring and exploring purposes are presented in [3].

The macros-instrument concept, which has been introduced in [4], allows the spatial discovery of an environment utilizing distributed sensing platforms. These change information using wireless network communication services. The platforms are heterogeneous, including all types of communication: satellite and terrestrial; fixed and mobile.

Additional information proved to be very useful for the traffic participants. It includes data regarding the current location and information about traffic in adjacent area, the finding of best routes to avoid congestions etc. The Vehicular Ad-hoc NETworks (VANET) are appropriate to offer relevant, supplementary information and to offer solutions for problems regarding pollution, 
traffic congestion, fuel saving. Hundreds of fuel litters and hours spent in traffic can be saved using VANET enabled vehicles. The VANETs' users benefit from useful information, applications, statistics and news, and also can give, from their locations, inside data regarding the traffic conditions.

Many studies and scenarios were developed to assess the feasibility and efficiency of VANET based applications and to test new protocols. Simulation software is widely used to design real world scenarios $[5,6,7,8]$.

In this context, the paper proposes RTMCS (Road Traffic Monitoring and Control System) as complex system, which integrates maps generation, mobility analysis, simulation and control structures for road traffic applications using VANET simulators and Web-based tools.

The flexibility and the modularity of the system, the specific adapted design of the assembly and of each component element allow relevant tests adapted to a great number of possible variants and particularizations, which can be included in three main categories, as follows:

- The improvement of the traffic conditions for a given infrastructure; it implies the modification of the existing traffic routes in conditions corresponding to situations in which jamming or critical traffic events occur and the finding of alternative solutions for congested and perturbed traffic; the solutions considered for the both categories of problems are based on the using of the dynamic traffic signs and of the road segments which belong to the existing infrastructure and are only partial used in normal conditions;

- The design of new infrastructures laying the emphasis on the choice, positioning and settings of the traffic control, on supervising and guidance structures, in accordance with real conditions. A special attention was given to the minimization of the travel time and generally to improvement of the traffic conditions;

- The corresponding setting of traffic control parameters during the design phases, including the imposition of some restrictions, the positioning of traffic signs and semaphores and the establishing of the duration of their signaling colors.

The system integrates Web based and advanced mobile communication technologies and facilitates the running and interpretation of the results of VANET simulations using various simulators (like VanetMobiSim [9, 10, 11] and UDAS [13, 14]). A special module based on Glomosim [12] is used for the analysis of the communication system. A complex component based on UDAS [13,14] integrates the traffic simulator, the decision and control elements. A special attention was given to real time analysis performed using UPPAAL [15, 19 and 20] and UPPAAL Tron $[16,17,18]$ tools.

This complex architecture ensures the compatibility and combines predefined components such as VANET, Ad-Hoc networks simulators and GlomoSim with new, special designed structures for simulation and control, RTMCS. The RTMCS system allows integration of new tools that can be used to analyze and to design the road traffic.

The proposed web based system can be seen as a tools configurator useful to monitor and control the road traffic.

\section{RTMCS System Architecture}

A multilevel, distributed architecture has been proposed for the RTMCS system, Fig. 1.

The system is composed of the following main components: the Database Server, where the received and the processed data are stored and RTMCS Application Server, where the main components of the RTMCS are running. The interest information can be accessed by users through a Web Server. The distributed RTMCS systems communicate in a secure manner through a Virtual Private Network. 
The software components of the RTMCS system are presented in Fig. 2.

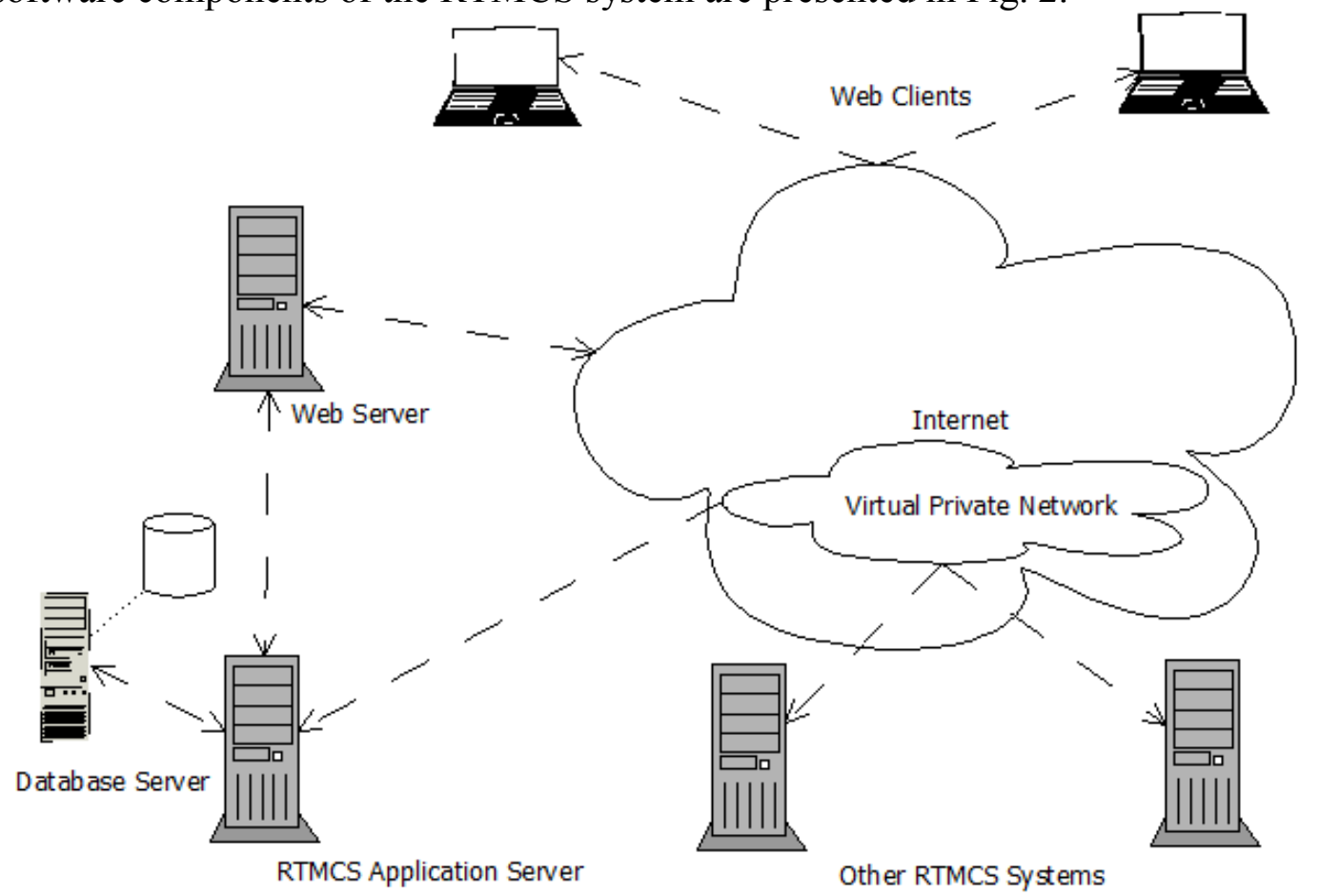

Figure 1. The RTMCS hardware architecture

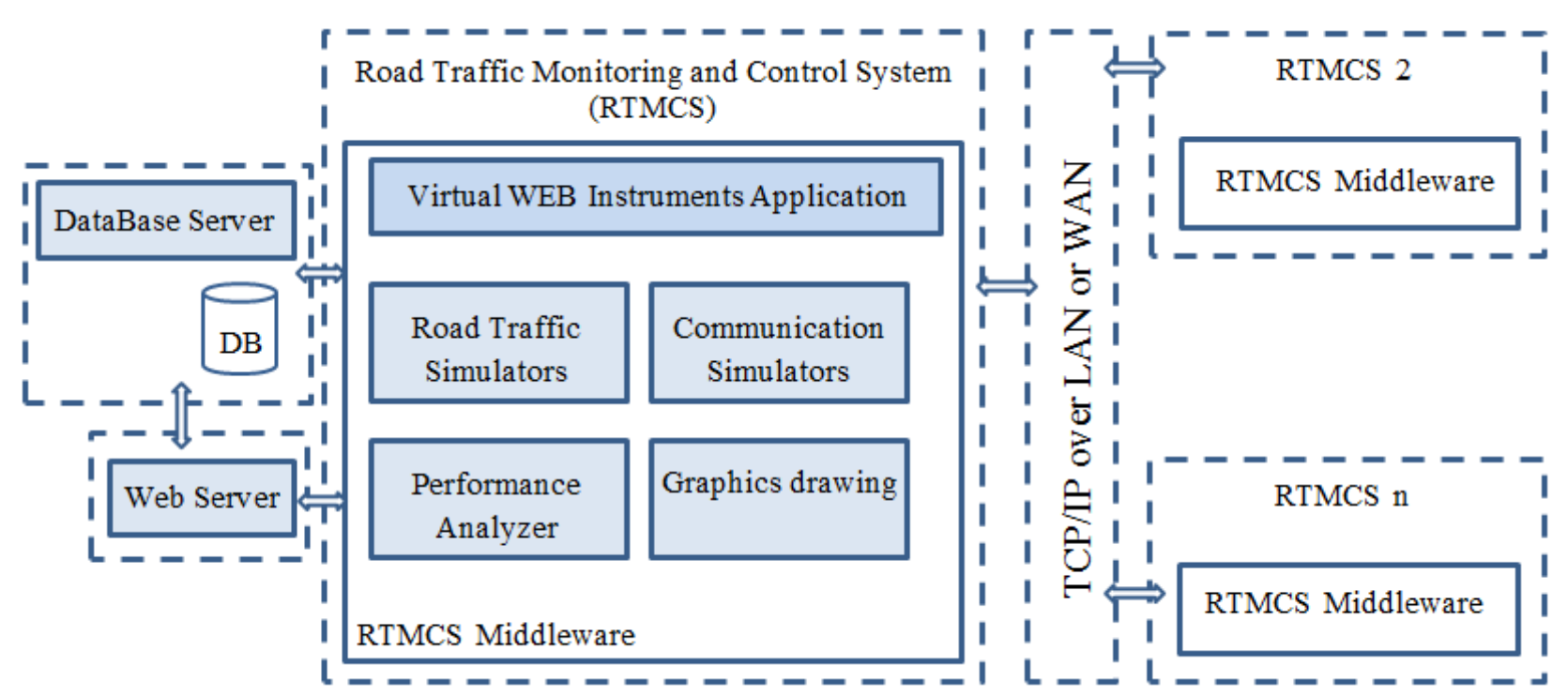

Figure 2. Software architecture of the RTMCS system

As stated in [21], a middleware application can be used as a support layer for wireless sensor network applications in order to meet challenges associated with this type of applications.

RTMCS middleware was developed in $\mathrm{C}++$, using the Wt framework, which offers for the UI (User Interface) a widget-centric solution.

The RTMCS middleware provides several services:

- Guarantees the data exchange between RTMCS nodes; a transparent access to simulation scenarios from remote RTMCS nodes is provided;

- Send notification for users or other applications;

- Provides authentication mechanisms;

- Provides functions for activation, deactivation of services.

This system handle errors which arise in the usual activities; on a lower layer the network layer ensures that fault and communication errors are detected and eliminated. The capacity of the system to manage extensions which imply the addition of new nodes is an important feature of the RTMCS. 
The functions of the main components of RTMCS are described as follows:

- To build scripts; the CMake scripts are separated into libraries;

- To creates a property dialog;

- The xml converters; to convert the UI model into xml and vice-versa;

- A server code with the database layer, the business validators and the simulations runners are also included into the presented structure;

- The utility library contains the logger and other useful tools.

A short description of the RTMCS components is given below.

Build system. CMake, the system generator is used for implementation of RTMCS. It is a crossplatform tool that takes as inputs the independent CMake files list, named CMakeLists.txt, included in each directory of a source tree. Within the files the components that make up a codebase are defined and are transformed by CMake into a specific platform build system (in RTMCS's case, the build system is based on GNU Make and $\mathrm{g}++$ ).

Client tier. The client tier encompasses the main loop method and all the specialized widgets and dialogues.

Map drawing. In the case in which the user chooses to define the topological space by drawing it, on the configuration page which contains the custom made PaintBrush widget the PaintBrushForm is added. This widget extends the Wt::WPaintedWidget class and renders as the $<$ canvas $>$ HTML 5 tag in the browser. On this canvas, the user can draw the topological space in accordance with the specified requirements. In order to draw a segment on the canvas, the user has to click once to set the first point and then, at different coordinates within the perimeter of the canvas to set the second point. The class will then render a segment between these two points.

XML converters. The $\mathrm{xml}$ folder of the source contains the implementation of the XML converters, the pugixml library being used. Three main components can be distinguished as follows: 1. The XML helper classes, which create an in-memory tree-like model representation of the XML. 2. A model of XML converter class, which takes the in-memory model and transforms it into a proper XML document.

3. A model of XML model converter class, which performs the reverse operation taking an XML document and transforms it into an in-memory representation.

The in-memory representation of the XML is created by the client tier. The converters change this model into XML documents which are then persisted into the database or added to the VanetMobiSim configuration file.

Server tier. The server tier is responsible for the configuration and the running of simulations and contains the classes used for the persisting of the configuration data. The persistence class relies heavily on Wt::Dbo library to connect to the database, create the working schema and perform the database operations. The configurator uses pugixml library to create the XML documents required by the simulators. The runners work in close relation with the classes that handle the configuration for each simulator.

Database. Three important classes can be mentioned:

- The entity classes: ConfigEntryEntity and ConfigurationEntity classes. They represent the two database tables. Each instance of any of these classes represents an entry in the table map; - The PersistenceManager class, a singleton that manages the database session and queries. 
The interaction between RTMCS Middleware's components is presented in Fig. 3.

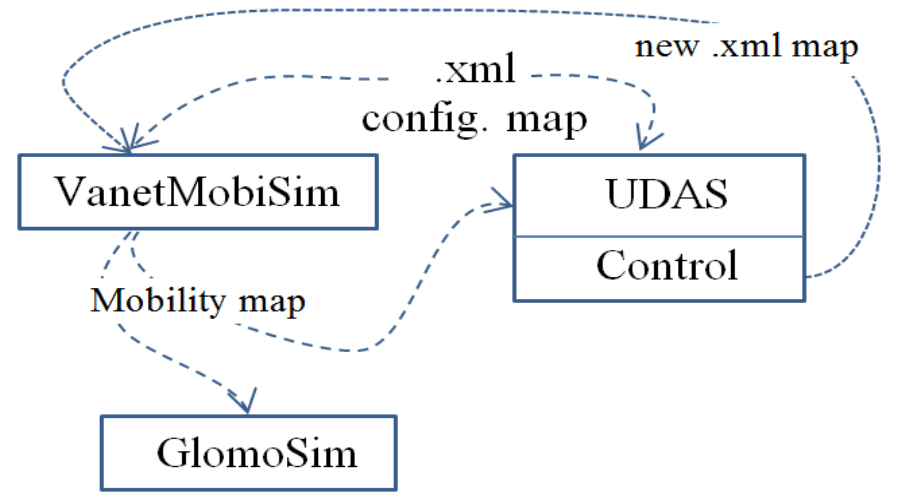

Figure 3. The interaction between RTMCS middleware's components

\section{General characteristics of the RTMCS system}

The complex browser based application facilitates for any user the design and simulation of VANETs applications using VanetMobiSim and GloMoSim. Such an application should fulfill the following broad requirements:

- The Creation of a VanetMobiSim .xml configuration;

- The selection of GloMoSim routing protocol configurations;

- The running of VanetMobiSim and GloMoSim simulators and the sending of the results in the requested format;

- The running of a real time simulation using UDAS;

- The running a real time analysis using UPPAAL and UPPAAL Tron for resource allocation;

- The saving of the configurations for later use.

VanetMobiSim was chosen for the traffic simulation due to its ease of use and high configurability.

For simulation purposes, VanetMobiSim receives as input an $\mathrm{xml}$ file that details the steps and the defined environment of the simulation. It is capable to simulating vehicles behavior patterns in different traffic conditions, taking into account different circulation signalization like traffic lights and imposed speed limits. From a geographic configuration point of view, VanetMobiSim accepts either a randomly generated road topology or a user defined environment.

GloMoSim is responsible for simulating the network routing protocols. The application offers the possibility to select among the following routing protocols: AODV (Ad-hoc On-Demand Distance Vector); BELLMAN-FORD; DSR (Dynamic Source Routing); LAR1 (Location-Aided Routing); WRP (Wireless Routing Protocol).

UDAS is a Java based implementation of a modular distributed simulator a large system built up of many interacting components of long road segments, short road segments and intersections, together with the boundary conditions at the edges of the configuration. Each component allows the representation of some random behavior during the evolution of the simulation.

UPPAAL is an integrated tool environment for modeling, validation and verification of real-time systems, modeled as networks of automata extended with data types (bounded integers, arrays, etc.) [15]. UPPAAL TRON is a testing tool, based on UPPAAL engine, suited for black-box conformance testing of timed systems, mainly targeted for embedded software commonly found in various controllers [16].

\section{The testing and simulation of the RTMCS Web Instruments Application}

Different simulation tools can be used for the testing and analyses of traffic scenarios. These also can design new road segments or modify existing traffic infrastructure. The RTMCS system allows the user to add new features, various aspects being taken into consideration. 
Simulation scenarios in which a variable number of participants were successively considered is presented below. First, the generated map is presented; then, the vehicles mobility is analyzed taking into consideration various constraints and road traffic features; finally, the wireless communication between traffic participants was analyzed.

The real time simulation of the traffic can be visualized using an updated and improved version of the simulator presented in $[13,14]$. This was integrated as a tool in the RTMCS system.

The performances of the modeled system were analyzed using UPPAAL and UPPAAL Tron, a different integrated tool. Configuration files in .xml format were generated; the output results of each tool were verified and validated using the implemented virtual tools. The interface file between two virtual tools was generated based on the configuration chosen by the user.

The web application has been successfully deployed on several x86 32 and 64 bit PCs. The minimal hardware requirements are: Pentium III 800 or equivalent; $256 \mathrm{MB}$ RAM; and at least $500 \mathrm{MB}$ of free space on HDD.

The map generated for simulation and analysis, Fig. 4, represents a part of a real, existing map. The simulation parameters were chosen as follows: a total area of $4 \mathrm{~km}^{2}(2 \times 2)$; two boulevards having two lanes in each direction; seven intersections, controlled by semaphores and several input and output gates; 200 vehicles for the first scenario and 20 vehicles for the second scenario; the average car length being $5.5 \mathrm{~m}$ and the average speed: $8.3 \mathrm{~m} / \mathrm{s}$.

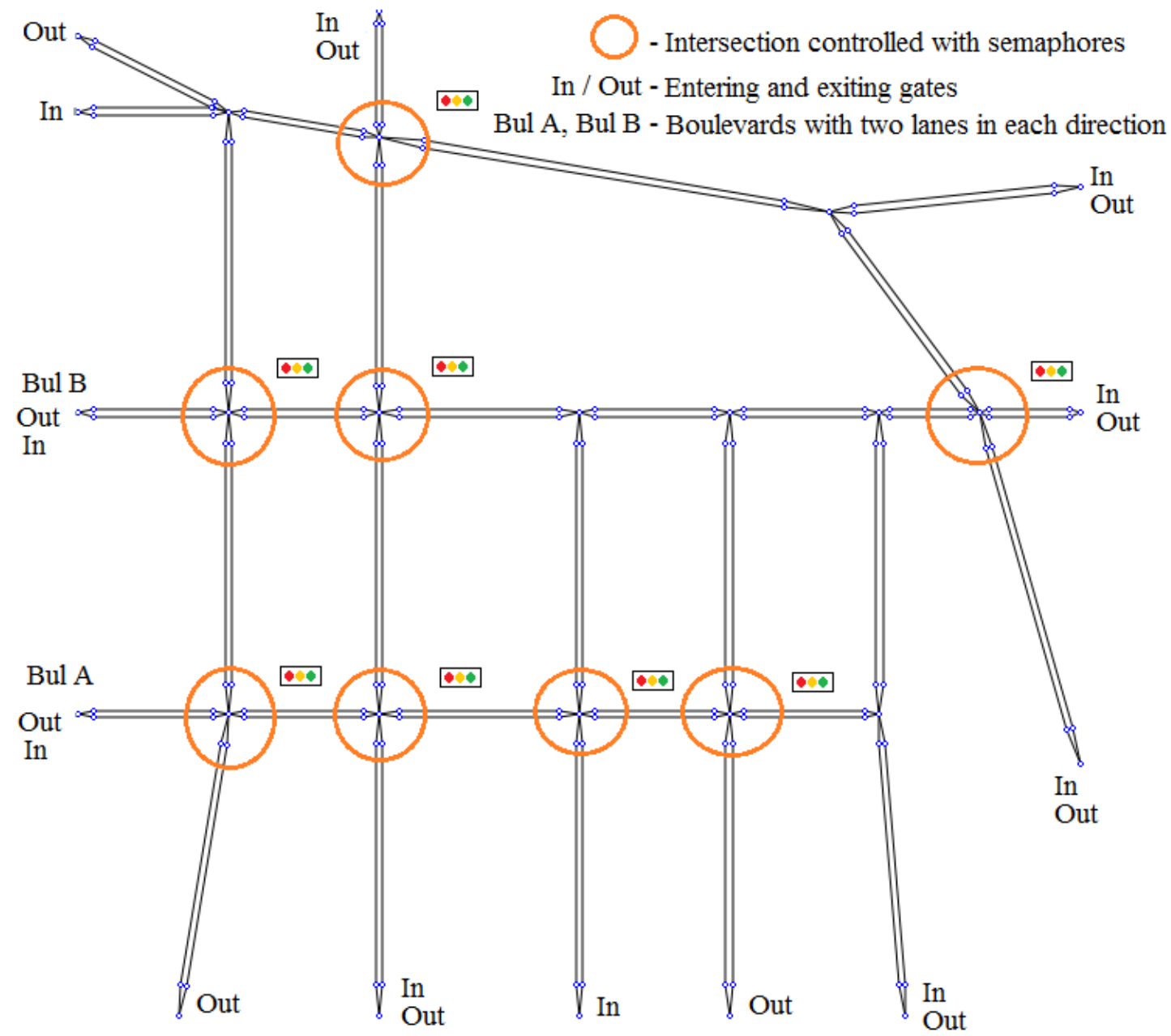

Figure 4. The simulated street map

For future analysis and comparison the system offers the possibility of saving different simulation. A ,suggestive" representation feature is implemented allowing the animation of different vehicles engaged in traffic as they move along the geographical topological space during a simulation. 
The Road Traffic Simulation. Each node and vertex of the considered simulated map is configured following the steps indicated in Fig. 5.

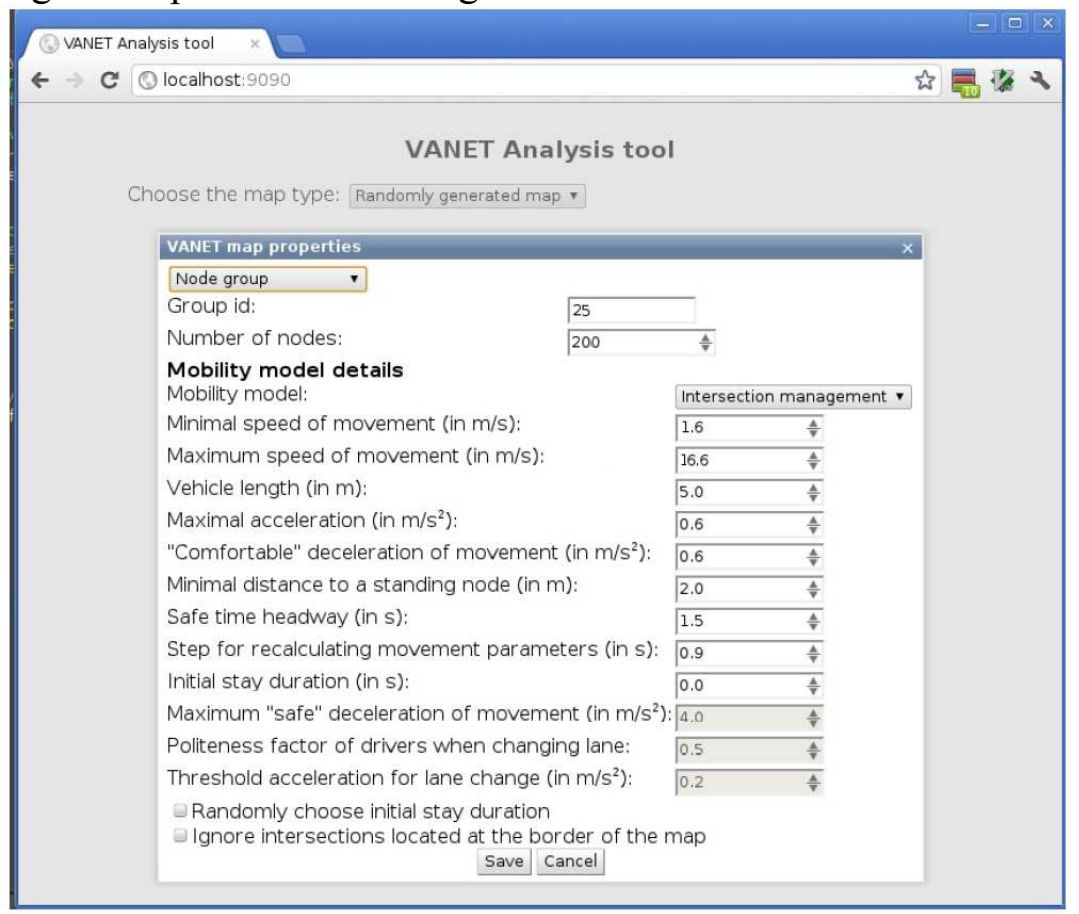

Figure 5. Traffic simulator property dialog for adding a node group

Some results of simulation are presented in Fig. 6.
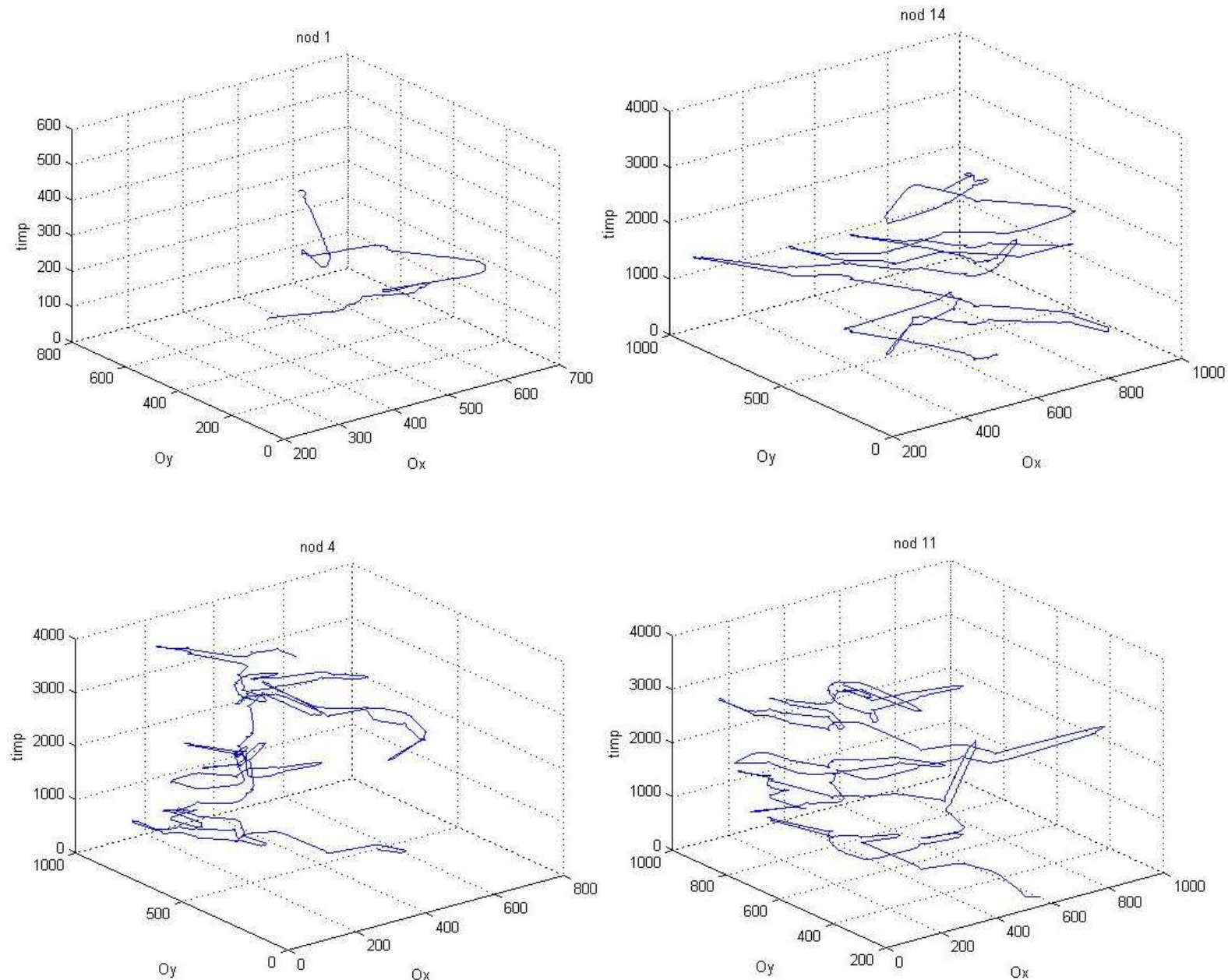

Figure 6. The graphical representation of the simulation result for four vehicles (random selection) 
The Communication Simulator. The communication simulator is used to test different scenarios of messages exchange among traffic participants, implying the mobility of the nodes and the achieving traffic data from static observers (traffic lights, counters, etc.).

The simulations were run on a PC having the following characteristics:

- 64-bit Windows 7 OS;

- CPU: Intel Core Duo, $2.20 \mathrm{GHz}$;

- RAM: 4.00 GB RAM;

- HDD: 50 GB of free space.

Parameters used for the first simulation scenario are presented in the second column of Table 1.

Table 1. Simulation scenarios parameters

\begin{tabular}{|l|c|c|}
\hline Parameters & Scenario I & Scenario II \\
\hline No. of mobile nodes (vehicles) & 200 & 20 \\
\hline $\begin{array}{l}\text { No. of static nodes (RTCMS servers, traffic lights, } \\
\text { counters) }\end{array}$ & $20(4$ servers) & 20 (4 servers) \\
\hline Map [km $\left.{ }^{2}\right]$ & $4(2 \times 2)$ & $4(2 \times 2)$ \\
\hline Min speed [m/s] & 5 & 5 \\
\hline Max speed [m/s] & 15 & 15 \\
\hline No. of crossroads controlled with traffic light & 8 & 8 \\
\hline Streets with several traffic lanes (2 lanes) & 4 & 4 \\
\hline Green light [s] & 10 & 10 \\
\hline
\end{tabular}

A second scenario in which the number of mobile nodes is smaller and the rest of the parameters are the same, is described in accordance with the data contained in the third column of Table 1.

In Table 2 the simulation results are presented.

Table 2. Simulation results

\begin{tabular}{|l|c|c|}
\hline Parameters & Scenario I & Scenario II \\
\hline RTCMS Server 1 - average end to end delay [s] & 0.075148 & 0.011161 \\
\hline RTCMS Server 2 - average end to end delay [s] & 0.303564 & 0.331957 \\
\hline RTCMS Server 3 - average end to end delay [s] & 0.008456 & 0.007552 \\
\hline RTCMS Server 4 - average end to end delay [s] & 0.283064 & 0.018896 \\
\hline Total execution time [s] & 80.1400 & 17.9220 \\
\hline
\end{tabular}

It must be mentioned that the final results are influenced by the chosen package routing algorithms. A comparison of results obtained using AODV, DSR and LAR protocols is presented in Fig. 7.

For the second level of the design of a new vehicular traffic topology, the new structure must be created in accordance with the urban and suburban traffic and with the existing infrastructure. The new network must encapsulated characteristics components having specific features such as:

- Traffic lights;

- Intersections;

- Drivers behavior;

- Obstacles;

- Traffic constraints;

- Streets topology;

- Urban and suburban topology. 


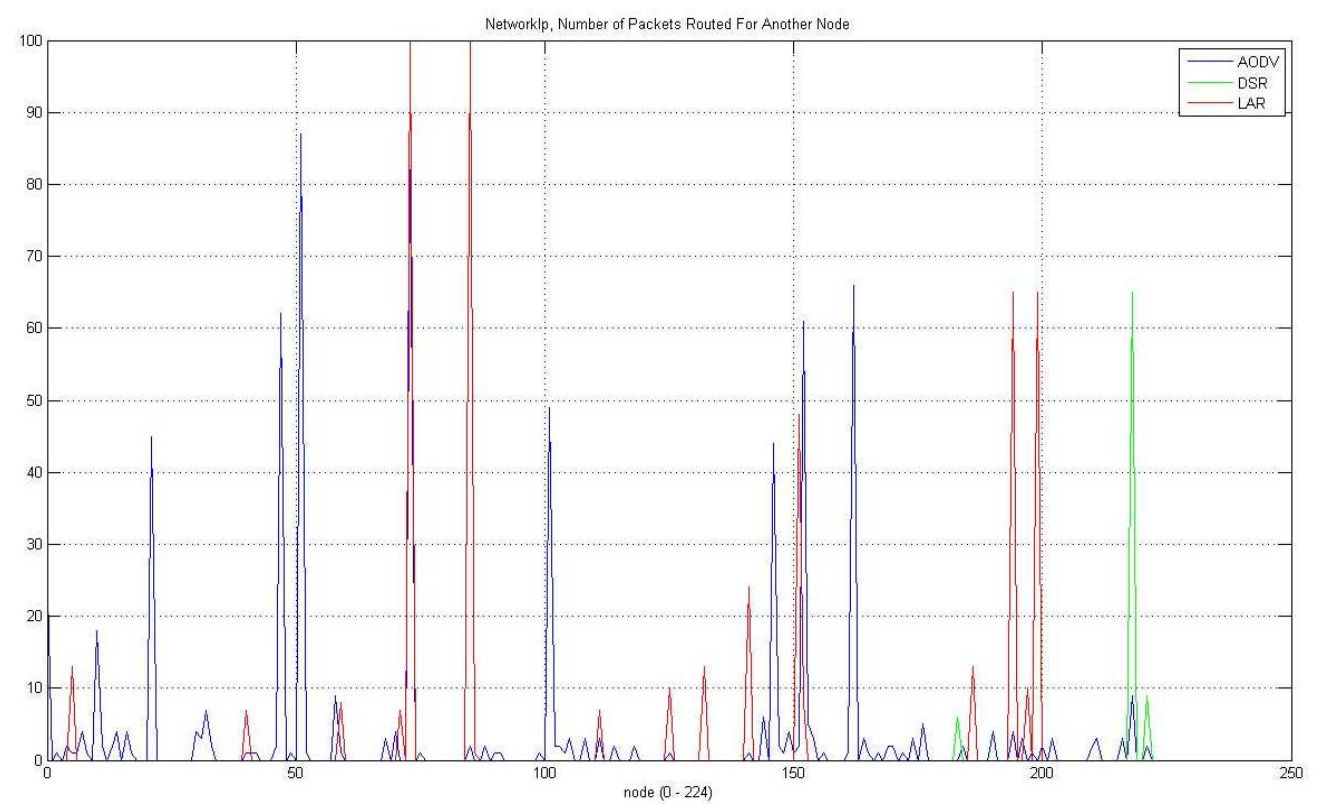

Figure 7. Number of package routed for a different node

\section{Conclusions}

A web based virtual instrumentation system, RTMCS, was proposed in order to offer support for the surveillance, control and monitoring of road networks. It integrates complex created components to validate a variety of road traffic models. RTMCS take into account real time constraints and include tools to simulate various traffic scenarios, communication technologies and it has a personalized user interface. It offers several advantages, one of the most important consisting of the possibility to choose different configuration and components using a web browser. The open structure of the system offers multiple possibilities to modify the simulation conditions (which include the modification of road infrastructure). The system was designed not only to offer flexibility, but also the advantages of multiple choices. Due to modularity and a great number of combinations among the possibilities offered by each module, various structures can be built, mapping representative cases of real situations.

UPPAAL model checker allows the analysis of the models that capture the dynamic behavior of systems. The program is particularly useful to analyze the behavioral properties, to detect possible deadlocks and to verify real time constraints but imply a relatively costly solution from the computational point of view.

The presented experiments and their corresponding results highlight the functionality of the configurable virtual web instrument concept. The open structure of the system allows the implementation of several road traffic routing strategies based on real time collected data and the extensions of RTMCS functions in accordance with different preferences and new necessities.

\section{Acknowledgements}

This paper was supported by the project "Development and support of multidisciplinary postdoctoral programs in major technical areas of national strategy of Research - Development Innovation" 4D-POSTDOC, contract no. POSDRU/89/1.5/S/52603, project co-funded by the European Social Fund through Sectorial Operational Program Human Resources Development 2007-2013. 


\section{References}

[1] C. Lin, Virtual detector in adaptive traffic signal control system based on support vector machine, Conference on Automation and Logistics, IEEE International, (2009) 961-965.

[2] H.F. Teng, M.J. Wang, C.M. Lin, An Implementation of Android-Based Mobile Virtual Instrument for Telematics Applications, Second International Conference on Innovations in Bio-inspired Computing and Applications IBICA, (2011), 306-308.

[3] K. A. Delin, S.P. Jackson, The Sensor Web: A New Instrument Concept. Presented at SPIE's Symposium on Integrated Optics, San Jose, CA, (2001), 4-16.

[4] K.A. Delin, The Sensor Web: A Macro-Instrument for Coordinated Sensing, Sensors Journal, 2(7), (2002), $270-285$.

[5] A. Hassan, VANET Simulation, School of Information Science, Computer and Electrical Engineering Halmstad University, 2009.

[6] A.L. Kun, I. Cassias, Vehicle Telematics: A Literature Review, Technical Report ECE.P54.2007.9, 2007.

[7] F.M. Pardon, Traffic Congestion Detection Using VANET, Florida Atlantic University Boca Raton, Florida, 2009.

[8] S. Jain, S. Taneja, D. Jain, Fuzzy logic based routing strategies in VANETs, Proceedings of the 3rd National Conference, India, (2009), 10-15.

[9] VanetMobiSim - Vehicular Ad hoc Network mobility extension to the CanuMobiSim framework, Institut Eurécom/Politecnico di Torino, 2006.

[10] VanetMobiSim : http://vanet.eurecom.fr/

[11] CanuMobiSim: http://canu.informatik.uni-stuttgart.de/mobisim/downloads/

[12] GloMoSim: http://pcl.cs.ucla.edu/projects/glomosim/academic/topsecret_download.html

[13] C. Avram, R. Boel, Distributed implementation of a heterogeneous simulation of urban road traffic, High Performance Computing \& Simulation Conference, HPC\&S'05, Riga, Latvia, (2005), 833-840.

[14] C. Avram, A. Aştilean, T. Leţia, Multi Agent Implementation of an Urban Road Traffic Advisor, High Performance Computing \& Simulation Conference (HPC\&S'06), Koln, Germany, (2006), 340-345.

[15] G. Behrmann, A. David, and K. G. Larsen, A Tutorial on UPPAAL 4.0, Updated November 28, 2006.

[16] K. G. Larsen, M. Mikucionis, B. Nielsen, UPPAAL Tron User Manual, CISS, BRICS, Aalborg University, Aalborg, Denmark, 2009.

[17] A. Hessel, K. G. Larsen, M. Mikucionis, Brian Nielsen, Paul Pettersson and Arne Skou. Testing Real-Time Systems Using UPPAAL, Formal Methods and Testing. Springer Berlin / Heidelberg. 2008.

[18] M. Mikucionis, UPPAAL TRON: Testing Real-time systems Online, MoDES project meeting. Sønderborg, Denmark, 2007.

[19] P. Ravn, J. Srba, S. Vighio. Modelling and Verification of Web Services Business Activity Protocol, Proceedings of the 17th International Conference on Tools and Algorithms for the Construction and Analysis of Systems, (2010), 357-371.

[20] A.P. Ravn, S. Vighio and J. Srba. A Formal Analysis of the Web Services Atomic Transaction Protocol with UPPAAL, Proceedings of the 4th International Symposium On Leveraging Applications of Formal Methods, Verification and Validation ISOLA, LNCS, SpringerVerlag, (2010), 579-593.

[21] S. Hadim, N. Mohamed, Middleware challenges and approaches for wireless sensor networks. IEEE Distributed Systems Online 7(3) (2009), 1-1.

[22] G. Lu, B. Krishnamachari, Minimum latency joint scheduling and routing in wireless sensor networks, Ad Hoc Networks, 5, (2007), 832-843.

[23] J.A. Stankovic, When Sensor and Actuator Networks Cover the World, ETRI Journal, 30(5), (2008), 627-633.

[24] I. Stojmenovic, Handbook of Sensor Networks, Algorithms and Architectures, Wiley Interscience, 49, 2005. 